\title{
Interaction-Aware Occupancy Prediction of Road Vehicles
}

\author{
Markus Koschi and Matthias Althoff
}

\begin{abstract}
A crucial capability of autonomous road vehicles is the ability to cope with the unknown future behavior of surrounding traffic participants. This requires using nondeterministic models for prediction. While stochastic models are useful for long-term planning, we use set-valued nondeterminism capturing all possible behaviors in order to verify the safety of planned maneuvers. To reduce the set of solutions, our earlier work considers traffic rules; however, it neglects mutual influences between traffic participants. This work presents the first solution for establishing interaction within set-based prediction of traffic participants. Instead of explicitly modeling dependencies between vehicles, we trim reachable occupancy regions to consider interaction, which is computationally much more efficient. The usefulness of our approach is demonstrated by experiments from the CommonRoad benchmark repository.
\end{abstract}

\section{INTRODUCTION}

It is commonly agreed that purely reactive controllers for collision avoidance only considering the current situation are insufficient for avoiding collisions in road traffic. Integrating a prediction of other traffic participants facilitates much better solutions [1].

Depending on the purpose of the vehicle motion planner or driving assistant system, different types of prediction are appropriate. For driving assistant systems, simple predictions only producing a single behavior are sufficient [2]-[6], since warnings are not necessarily safety-critical. However, for long-term planning of automated vehicles, simple predictions are insufficient, since they do not explicitly consider the growing uncertainty when one increases the prediction horizon. Stochastic approaches account for this shortcoming [7]-[11]. To guarantee safe movement, however, one cannot rely on stochastic approaches, since ensuring safety or a very small crash probability (around $10^{-10}$ for a $5 \mathrm{~s}$ prediction horizon) is necessary in order to obtain motions which are superior to those of humans. Such small probabilities are difficult to verify, so we propose set-based predictions as developed in our previous work [12], [13]. Set-based prediction, based on models with uncertain yet bounded inputs and parameters, contains all possible movements of traffic participants.

Clearly, set-based prediction considering all possible behaviors can block unnecessarily large sections of a road network for the motion planner. To manage this issue, we predict behaviors that comply with traffic rules only, which can be individually deactivated in case of violation. In addition, one can restrict the prediction horizon by computing fail-safe

Markus Koschi and Matthias Althoff are with the Department of Informatics, Technical University of Munich, 85748 Garching, Germany. \{markus.koschi, matthias.althoff\}@tum.de

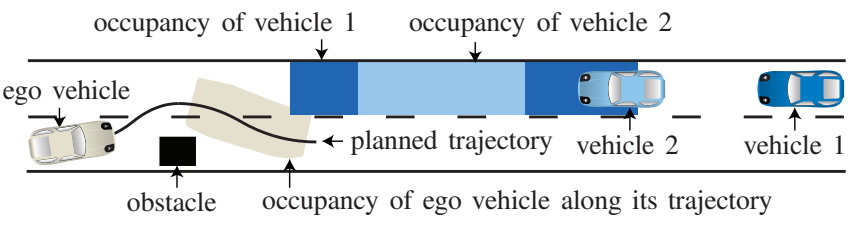

(a) Planned trajectory is unsafe without considering interaction.

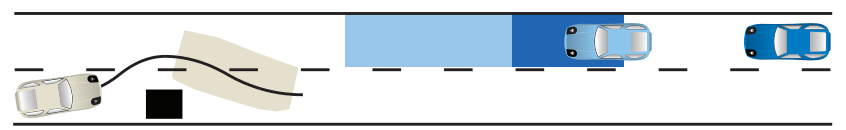

(b) Planned trajectory can be verified as safe when considering interaction.

Fig. 1. Occupancies of interacting vehicles for a selected time interval.

maneuvers [14]. However, we have not yet exploited mutual influences between traffic participants to improve the quality of the prediction.

One possibility for considering interaction would be to use a concrete model of dependencies. However, such models are typically unknown [15] and result in large combined systems, which are hard to analyze. Instead, we consider interaction between traffic participants on a more abstract level: e.g. when two cars drive in a lane as shown in Fig. 1, the maximum reachable position of the following vehicle 1 can never be greater than the maximum reachable position of the leading vehicle 2. Fig. 1(a) shows the occupancies of both vehicles, i.e. the region they can occupy in the selected time interval (see Def. 9 later), without considering their interaction. When taking this into account, the region occupied by vehicle 1 can be shortened for all consecutive time intervals such that it no longer reaches in front of the occupancy of vehicle 2, as shown in Fig. 1(b).

There is only very little work considering interactions between traffic participants for prediction, as pointed out in [1]. For single behavior prediction, one can assume that other vehicles avoid collisions and thus penalize the trajectories which result in a collision [16], [17]. In terms of stochastic prediction, the work in [18] considers interaction by adjusting the acceleration and lane-change behavior of following vehicles. Since modeling the pairwise dependencies between traffic participants grows with the number of entities, one can reduce the complexity by assuming unidirectional influence [19], [20]. Instead of considering the dependencies pairwise, the authors of [21] model mutual influences as a function of the local situational context. Based on [21], a fully probabilistic model is presented in [22]. The work in [23] presents experience-based data on the interaction between the ego and surrounding vehicles during lane changes. In order to 
consider interaction in situation assessment, one can compute an interaction-aware joint probability distribution [24] or detect conflicting intentions at intersections by comparing what vehicles intend to do with what they are expected to do [25].

This work is the first which incorporates interaction into set-based prediction of other traffic participants. Our paper is organized as follows: After providing relevant definitions in Sec. II, we introduce set-based prediction in Sec. III. Sec. IV defines our concept for considering interaction and describes our algorithm for removing unreachable occupancy regions. Numerical experiments are presented in Sec. V and discussed in Sec. VI.

\section{PRELIMINARIES}

\section{A. Road Network}

Our road network model is composed by lanelets [26], which are atomic, interconnected, and drivable road segments:

Definition 1 (Lanelets [26]): A lanelet is defined by its left and right bound, where each bound is represented by an array of points (a polyline), as shown in Fig. 2. The driving direction of a lanelet is implicitly defined by its left and right bound.

To represent the road network as a directed graph, we introduce relations between two lanelets: successor, left, and right.

Definition 2 (Lanes): We define lanes as the union of lanelets which are longitudinally adjacent, i.e. are successors of each other.

Note that a lanelet which has multiple successors, as in the case of road forks, becomes an element of multiple lanes (e.g. see lanelet 2 in Fig. 2).

Definition 3 (Merging Lanes): Two lanes are merging into one lane, if they are constructed from distinct lanelets which eventually have a common successor lanelet (e.g. see Fig. 5). The geometric condition for merging lanes is that the start points of the two lanes must be different and their end points must be equal (see Fig. 2 for the definition of these points).

Definition 4 (Current Lanes of a Vehicle): The current lanes of a vehicle are defined as all lanes in which the

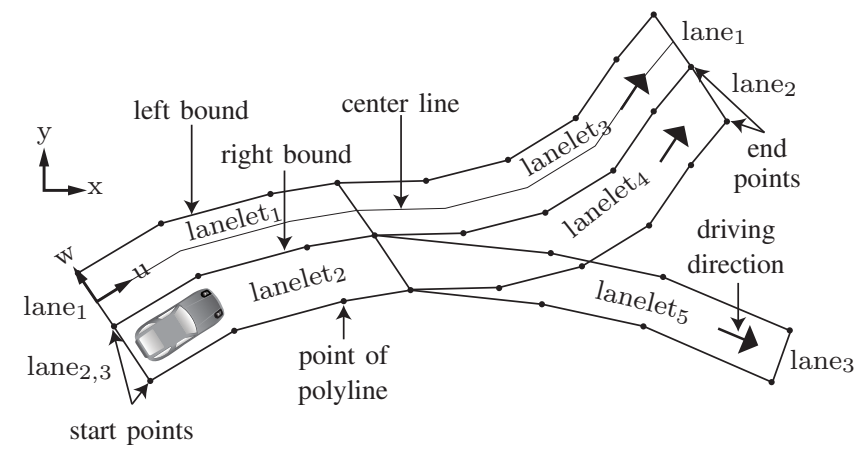

Fig. 2. Our road network is modeled by lanelets and lanes. vehicle is currently positioned (e.g. in Fig. 2, lane 2 and lane $_{3}$ are the current lanes of the vehicle).

In addition to the Cartesian space in world coordinates xy, we require a lane coordinate system uw:

Definition 5 (Curvilinear Lane Coordinate System): A curvilinear lane coordinate system uw is defined for each lane such that the $\mathrm{u}$-axis is parallel to the center line of the lane and the $\mathrm{w}$-axis is perpendicular to $\mathrm{u}$, as shown in Fig. 2. The origin of the lane coordinate system is in the start point of each center line, and the positive $\mathrm{u}$-axis points in the driving direction. The $\mathrm{u}$-coordinate of a point $p$ in lane $l_{i}$ is denoted by $\mathrm{u}_{p}^{l_{i}}$.

Definition 6 (Front-Most and Rear-Most Point): For a set of points $\mathcal{P}$, the point with the maximum u-coordinate in lane $l_{i}$ is defined as

$$
\max \left(\mathrm{u}_{\mathcal{P}}^{l_{i}}\right):=\max \left(\mathrm{u}_{p}^{l_{i}} \mid p \in \mathcal{P}\right) .
$$

The rear-most point of $\mathcal{P}$ in $l_{i}, \min \left(\mathrm{u}_{\mathcal{P}}^{l_{i}}\right)$, is defined analogously.

For the sake of clarity, we omit the lane's notation by using only $\mathrm{u}_{p}$ or $\mathrm{u}_{\mathcal{P}}$ if the point or the set of points is defined in only one lane.

\section{B. Occupancy of a Vehicle}

The dynamics of a vehicle can be described by the differential equation

$$
\dot{x}(t)=f(x(t), u(t)),
$$

where $x \in \mathbb{R}^{n}$ is the state and $u \in \mathbb{R}^{m}$ is the input. The possible initial states and the possible inputs are bounded by sets: $x(0) \in \mathcal{X}_{0}, \forall t: u(t) \in \mathcal{U}$.

Definition 7 (Reachable Set): The reachable set $\mathcal{R} \subseteq \mathcal{X}$ of (1) is the set of states which are reachable at a certain point in time $r$ from a set of initial states $\mathcal{X}^{0}$ at time $t_{0}$ and subject to the set of inputs $\mathcal{U}$ :

$$
\mathcal{R}(r)=\left\{\int_{0}^{r} f(x(t), u(t)) d t \mid x(0) \in \mathcal{X}^{0}, \forall t: u(t) \in \mathcal{U}\right\} .
$$

Furthermore, we introduce a relation from a state vector $x$ to the Cartesian coordinate system xy:

Definition 8 (Relation to Cartesian Space): The operator state $2 \operatorname{occ}(x)$ relates the state of a vehicle to the set of points in Cartesian space occupied by the vehicle (including its dimensions) as

$$
\operatorname{state} 2 \operatorname{occ}(x): \mathcal{X} \rightarrow \mathcal{P}\left(\mathbb{R}^{2}\right),
$$

where $\mathcal{P}\left(\mathbb{R}^{2}\right)$ is the power set of $\mathbb{R}^{2}$. Given a set of states $\mathcal{X}$, the relation is defined as $\operatorname{state} 2 \operatorname{occ}(\mathcal{X}):=$ $\{$ state $2 \operatorname{occ}(x) \mid x \in \mathcal{X}\}$.

Definition 9 (Over-approximative Occupancy Set):

Based on Def. 7 and Def. 8, the occupancy set $\mathcal{O}(t)$ over-approximates the set of occupied points in Cartesian space which are reachable by the vehicle:

$$
\forall t: \mathcal{O}(t) \supseteq \operatorname{state} 2 \operatorname{occ}(\mathcal{R}(t)) .
$$

We can use over-approximative occupancy sets to describe the unknown future behavior of vehicles. 


\section{Set-BASEd PREDiction}

The set of future occupancies according to Def. 9 can be obtained with set-based prediction [12]. Using reachability analysis, we predict occupancies for consecutive time intervals as shown in Fig. 3, where we use polygons as set representation. Given the predicted occupancies of other vehicles and the occupancy of the ego vehicle along its planned trajectory, the planned trajectory can be verified as safe [27]: If none of the computed occupancies intersects with the occupancy of the ego vehicle for all points in time, one can guarantee that the ego vehicle does not cause a collision.
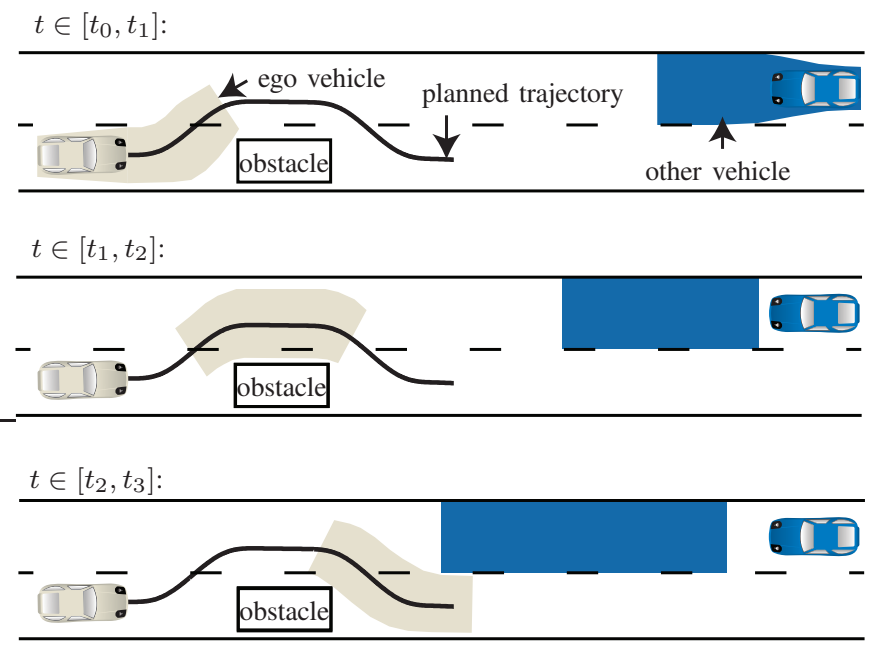

Fig. 3. Snapshots of the predicted occupancy of the other vehicle for selected consecutive time intervals.

Set-based prediction is designed to verify motion plans of short time horizons. Due to the full consideration of uncertainties, the future occupancy of other vehicles grows over time and thus limits the solution space for the ego vehicle. For this reason, we suggest performing trajectory planning for two time horizons in parallel [12]: While non-formal prediction techniques help to find long-term motion plans, set-based occupancy prediction can be used to guarantee the safety of short-term motion plans.

We compute over-approximative occupancies including all possible behaviors under given constraints, which are listed in Tab. I. All assumptions are taken from [13] and are either physical constraints $\left(C_{a_{\max }}\right.$ and $\left.C_{\text {engine }}\right)$ or a formalization of the Vienna Convention on Road Traffic [28], [29]. Please note that we deactivate constraints individually during online execution if traffic rules are violated. For more details on our constraint management, please see [13].

\section{INTERACTIONS BETWEEN VEHICLES}

Since vehicles share the same road, their presence and actions constantly influence other vehicles. As an example, Fig. 1 shows a traffic scenario in which considering interaction is important. While the ego vehicle plans an overtaking maneuver similar to the situation in Fig. 3, it has to consider two oncoming vehicles with different initial velocities.
TABLE I

VEHICLE CONSTRAINTS.

\begin{tabular}{|c|c|}
\hline Constraint & Description \\
\hline$C_{a_{\max }}$ & Maximum absolute acceleration is limited by $a_{\max }$. \\
\hline$C_{v_{\max }}$ & $\begin{array}{l}\text { Positive longitudinal acceleration is stopped when } \\
\text { a parameterized speed } v_{\max } \text { is reached. }\end{array}$ \\
\hline$C_{\text {engine }}$ & $\begin{array}{l}\text { Above a parameterized speed } v_{S} \text {, acceleration in } \\
\text { the driving direction is } a_{\text {long }}=a_{\max } \frac{v_{S}}{v} \text {, which } \\
\text { models limited engine power. }\end{array}$ \\
\hline$C_{\text {back }}$ & Driving backwards in a lane is not allowed. \\
\hline$C_{\text {lane }}$ & $\begin{array}{l}\text { Leaving the lane is forbidden. Changing lanes is } \\
\text { only allowed if the new lane has the same } \\
\text { driving direction as the previous one. }\end{array}$ \\
\hline
\end{tabular}

The following vehicle 1 moves faster than the preceding vehicle 2 . Hence, the independently predicted occupancy of vehicle 1 is larger than the occupancy of vehicle 2 , as shown in Fig. 1(a). For a certain time interval, the ego vehicle might crash into vehicle 1 when following its planned trajectory. However, vehicle 1 cannot reach the part of its occupancy where it ranges in front of the occupancy of vehicle 2 , since it cannot surpass vehicle 2 . When considering the interactions, we can remove the unreachable region and thus the plan of the ego vehicle can be verified as safe (see Fig. 1(b)).

Set-based occupancy prediction has neglected dependencies between traffic participants so far. In this section, we describe our extension to consider the interactions between vehicles. Our rule-based approach focuses on two-lane roads with only one lane per driving direction. We do not include roads with multiple lanes per driving direction, since vehicles can easily overtake others in the left and right lanes, as demonstrated later in Sec. V. Thus, only small regions are not reachable. For set-based prediction, which must include all reachable occupancies (see Def. 9), considering interactions in multi-lane scenarios is not beneficial. Instead, we incorporate dependencies between vehicles which are in the same lane of two-lane roads, i.e. the considered vehicles are either in the same current lane or in merging lanes, as described later in Sec. IV-B. Since we only handle vehicles in the same lane, it is sufficient to compare vehicles pairwise to consider their interaction.

\section{A. Overall Algorithm}

Alg. 1 gives an overview of the computation steps to consider interaction. From the set-based prediction, we require the independently computed occupancies of all vehicles for all time intervals $\tau_{k}$ from the initial time $t_{0}$ until the prediction horizon $t_{f}$, where $\tau_{k}=\left[t_{k}, t_{k+1}\right]$ with a time step size of $\Delta t=t_{k+1}-t_{k}$. First, we sort all vehicles which are in the same lane based on their initial position (see line 1 of Alg. 1 and Sec. IV-B). The returned list [v] contains the pairwise sorted vehicles as a tuple $\left(\mathrm{v}_{i}, \mathrm{v}_{i+1}\right)^{\left[t_{s_{i}}, t_{e_{i}}\right]}$, which represents the fact that $\mathrm{v}_{i}$ is behind $\mathrm{v}_{i+1}$ in the time interval $\left[t_{s_{i}}, t_{e_{i}}\right]$. Please note that due to road forks and merging lanes, the order of the vehicles in each tuple is only valid 
from the specified start time $t_{s_{i}}$ to the end time $t_{e_{i}}$, as explained in more detail later. An example of the list [v] is

$$
\begin{aligned}
{[\mathrm{v}]=} & {\left[\left(\mathrm{v}_{1}, \mathrm{v}_{2}\right)^{\left[t_{s_{1}}, t_{e_{1}}\right]},\left(\mathrm{v}_{3}, \mathrm{v}_{4}\right)^{\left[t_{s_{3}}, t_{e_{3}}\right]}, \ldots,\right.} \\
& \left.\left(\mathrm{v}_{n}, \mathrm{v}_{n+1}\right)^{\left[t_{s_{n}}, t_{e_{n}}\right]}\right] .
\end{aligned}
$$

Second, we consider the interaction of all vehicle pairs $\left(\mathrm{v}_{i}, \mathrm{v}_{i+1}\right)^{\left[t_{s_{i}}, t_{e_{i}}\right]}$ from front to back in each lane (see line 2 to 4 of Alg. 1). The occupancy of all following vehicles $\mathrm{v}_{i}$ is trimmed $\forall \tau_{k} \subseteq\left[t_{s_{i}}, t_{e_{i}}\right]$ such that unreachable areas are removed, as described in Sec. IV-C. In the following, we denote an element of [v] without loss of generality by $\left(\mathrm{v}_{1}, \mathrm{v}_{2}\right)^{\left[t_{s}, t_{e}\right]}$ and omit further indices for the sake of clarity.

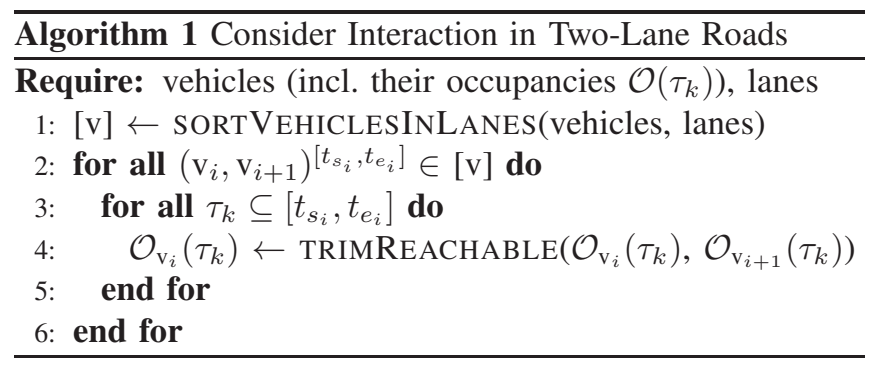

\section{B. Sort Vehicles in the Same Lane}

Through a pairwise comparison of all vehicles, the function SORTVEHICLESINLANES() of Alg. 1 sorts vehicles in their lanes in ascending $\mathrm{u}$-coordinates and returns them in the list [v]. If we cannot guarantee that one vehicle will precede another due to the growing uncertainty in the prediction, this vehicle pair is omitted in [v]. When sorting, we distinguish three different cases:

1) Sort Vehicles in the Same Current Lane: Two vehicles are in the same current lane if they are in only one current lane (see Def. 4) and this lane is the same for both vehicles. Then, their order is unambiguously given by their $\mathrm{u}$-coordinates in the lane coordinate system (see Def. 5). As an example, Fig. 1 shows the sorted vehicles 1 and 2, which can be added to $[\mathrm{v}]$ as $\left(\mathrm{v}_{1}, \mathrm{v}_{2}\right)^{\left[t_{0}, t_{f}\right]}$ since $\mathrm{u}_{\mathrm{v}_{1}}<\mathrm{u}_{\mathrm{v}_{2}}$.

2) Sort Vehicles in Forking Lanes: In the case of road forks, where more than one current lane is identical, we can also sort two vehicles until their reachable occupancies split onto two different lanes after the road fork. Since the assumption of one lane per driving direction is invalid after road forks, we can no longer guarantee that one vehicle will precede another. To determine the time until we can sort vehicles in forking lanes, we introduce the point $p_{\text {fork }}$ as the intersection of the corresponding lane bounds of the bifurcating lanes, as shown in Fig. 4.

Definition 10 (Not Passed the Lane Fork): We formulate the predicate NOT_PASSED_FORK $\left(\mathcal{O}_{\mathrm{v}_{2}}, p_{\text {fork }}, t\right)$ using firstorder logic:

$$
\begin{gathered}
\text { NOT_PASSED_FORK }\left(\mathcal{O}_{\mathrm{v}_{2}}, p_{\text {fork }}, t\right) \Leftrightarrow \\
\max \left(\mathrm{u}_{\mathcal{O}_{\mathrm{v}_{2}}}^{l_{1}}(t)\right) \leq \mathrm{u}_{p_{\text {fork }}}^{l_{1}} \wedge \max \left(\mathrm{u}_{\mathcal{O}_{\mathrm{v}_{2}}}^{l_{2}}(t)\right) \leq \mathrm{u}_{p_{\text {fork }}}^{l_{2}} .
\end{gathered}
$$

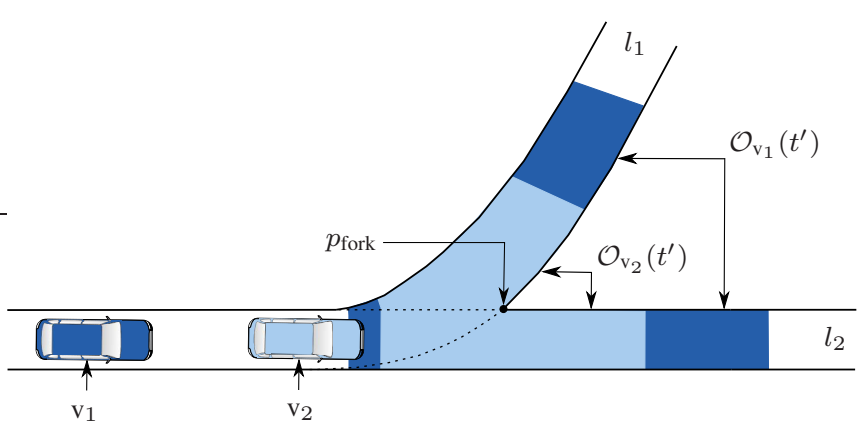

Fig. 4. The vehicles $\mathrm{v}_{1}$ and $\mathrm{v}_{2}$ cannot be unambiguously sorted for $t \geq t^{\prime}$, since $\mathcal{O}_{\mathrm{v}_{2}}\left(t^{\prime}\right)$ splits after the road fork.

It evaluates to true at time $t$ if the front-most point of $\mathcal{O}_{\mathrm{v}_{2}}(t)$ (see Def. 6) has not passed $p_{\text {fork }}$ in both bifurcating lanes $l_{1}$ and $l_{2}$.

Definition 11 (Time until the Lane Fork): We define $t_{\text {fork }}$ as the latest time at which the predicate NOT_PASSED_FORK $\left(\mathcal{O}_{\mathrm{v}_{2}}, p_{\text {fork }}, t\right)$ still evaluates to true:

$t_{\text {fork }}:=\max _{T \in \mathbb{R}}\left(\forall t: t \leq T:\right.$ NOT_PASSED_FORK $\left.\left(\mathcal{O}_{\mathrm{v}_{2}}, p_{\text {fork }}, t\right)\right)$.

Proposition 1 (Precedence in Forking Lanes): $\forall t: \tilde{t} \leq$ $t \leq t_{\text {fork }}$, vehicle $\mathrm{v}_{1}$ cannot precede vehicle $\mathrm{v}_{2}$ no matter what vehicle $\mathrm{v}_{2}$ is doing, where $\tilde{t}$ is some time within $\left[t_{0}, t_{\text {fork }}[\right.$.

Proof: If NOT_PASSED_FORK $\left(\mathcal{O}_{\mathrm{v}_{2}}, p_{\text {fork }}, t\right)$ evaluates to true (see Def. 10), vehicle $\mathrm{v}_{1}$ cannot precede vehicle $\mathrm{v}_{2}$ at time $t$, since they are still in the same current lane. In combination with Def. 11, Prop. 1 follows. The uncertainty of $\tilde{t}$ originates from the unspecified road network traversed before reaching the road fork.

Thus, we can sort vehicles in forking lanes analogously to vehicles in the same current lane (see previous paragraph), but only in the time interval $t_{s}=\tilde{t}$ to $t_{e}=t_{\text {fork }}$.

3) Sort Vehicles in Merging Lanes: If two vehicles are not yet in the same current lane, but their current lanes are merging (see Fig. 5), we also consider their interaction. All merging lanes can be detected by evaluating all pairs of lanes according to Def. 3. In order to argue about the order of vehicles in merging lanes, we must first define some distances, which are shown in Fig. 5. The distance along the $\mathrm{u}$-axis of lane $l_{2}$ between the rear-most point of $\mathcal{O}_{\mathrm{v}_{2}}(t)$ and the intersection point of the merging lanes $p_{\text {merge }}$ is defined as

$$
d_{\text {merge }}(t)=u_{p_{\text {merge }}}^{l_{2}}-\min \left(u_{\mathcal{O}_{\mathrm{v}_{2}}}^{l_{2}}(t)\right) .
$$

After describing the intersection of the occupancy of $v_{1}$ with the lane $l_{2}$ in which the other vehicle $v_{2}$ is positioned as

$$
\mathcal{O}_{\mathrm{v}_{1}}^{l_{2}}(t):=\mathcal{O}_{\mathrm{v}_{1}}(t) \cap l_{2},
$$

the distance between the front-most point of $\mathcal{O}_{\mathrm{v}_{1}}^{l_{2}}(t)$ and the rear-most point of $\mathcal{O}_{\mathrm{v}_{2}}(t)$ in $l_{2}$ can be defined as

$$
d_{\text {bounds }}(t)=\max \left(\mathrm{u}_{\mathcal{O}_{\mathrm{v}_{1}}^{l_{2}}}^{l_{2}}(t)\right)-\min \left(\mathrm{u}_{\mathcal{O}_{\mathrm{v}_{2}}}^{l_{2}}(t)\right) \text {. }
$$

Please note that both $d_{\text {merge }}(t)$ and $d_{\text {bounds }}(t)$ can be negative. 


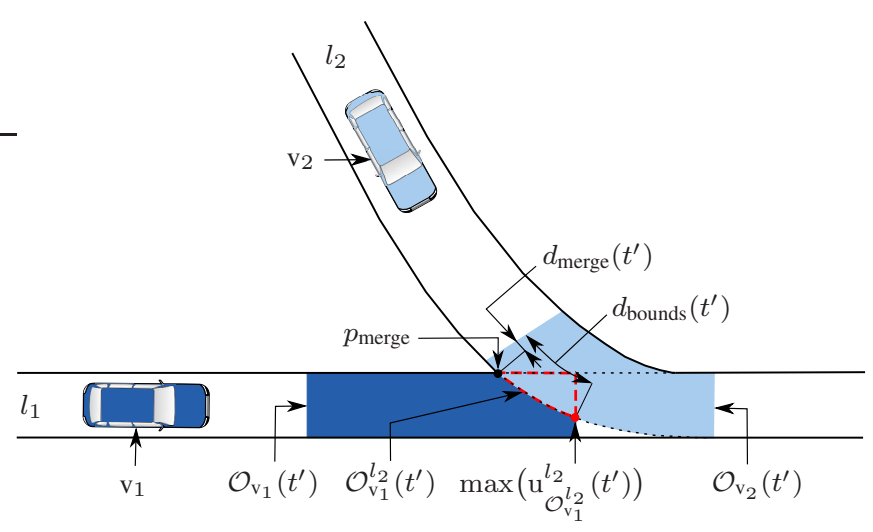

Fig. 5. The vehicles $\mathrm{v}_{1}$ and $\mathrm{v}_{2}$ in the merging lanes can be sorted at $t^{\prime}$, since $v_{1}$ cannot precede $v_{2}$ no matter what $v_{2}$ is doing.

Definition 12 (Passed the Lane Merge): We define the predicate PASSED_MERGE $\left(\mathcal{O}_{\mathrm{v}_{1}}, \mathcal{O}_{\mathrm{v}_{2}}, p_{\text {merge }}, t\right)$ as

$$
\begin{gathered}
\operatorname{PASSED} \text { MERGE }\left(\mathcal{O}_{\mathrm{v}_{1}}, \mathcal{O}_{\mathrm{v}_{2}}, p_{\text {merge }}, t\right) \Leftrightarrow \\
d_{\text {merge }}(t)<\text { length }_{\mathrm{v}_{2}} \wedge \\
\left(\mathcal{O}_{\mathrm{v}_{1}}^{l_{2}}(t)=\emptyset \vee d_{\text {bounds }}(t)<\text { length }_{\mathrm{v}_{2}}\right),
\end{gathered}
$$

where length $\mathrm{v}_{2}$ denotes the length of the vehicle's enclosing rectangle.

Definition 13 (Time after the Lane Merge): We introduce $t_{\text {merge }}$ as the earliest time at which PASSED_MERGE $\left(\mathcal{O}_{\mathrm{v}_{1}}, \mathcal{O}_{\mathrm{v}_{2}}, p_{\text {merge }}, t\right)$ evaluates to true:

$$
\begin{aligned}
t_{\text {merge }}:=\min _{T \in \mathbb{R}}(\forall t: t \geq T: \\
\text { PASSED_MERGE } \left.\left(\mathcal{O}_{\mathrm{v}_{1}}, \mathcal{O}_{\mathrm{v}_{2}}, p_{\text {merge }}, t\right)\right) .
\end{aligned}
$$

Proposition 2 (Precedence in Merging Lanes):

$\forall t: t_{\text {merge }} \leq t \leq \hat{t}$, vehicle $\mathrm{v}_{1}$ cannot precede vehicle $\mathrm{v}_{2}$ no matter what $\mathrm{v}_{2}$ is doing; $\left.\left.\hat{t} \in\right] t_{\text {merge }}, t_{f}\right]$.

Proof: Vehicle $\mathrm{v}_{1}$ can precede $\mathrm{v}_{2}$ at time $t$, either if $\mathrm{v}_{2}$ has not passed $p_{\text {merge }}$ but $v_{1}$ has, or if $d_{\text {bounds }}(t) \geq$ length $_{\mathrm{v}_{2}}$. Thus at $t^{\prime}$, if $d_{\text {merge }}\left(t^{\prime}\right)<$ length $_{\mathrm{v}_{2}}, \mathrm{v}_{2}$ certainly passed the intersection point $p_{\text {merge }}$. If, in addition, either $\mathcal{O}_{\mathrm{v}_{1}}^{l_{2}}\left(t^{\prime}\right)=\emptyset$ (i.e. $\mathrm{v}_{1}$ has not passed $\left.p_{\text {merge }}\right)$ or $d_{\text {bounds }}\left(t^{\prime}\right)<$ length $_{\mathrm{v}_{2}}$, the predicate PASSED_MERGE $\left(\mathcal{O}_{\mathrm{v}_{1}}, \mathcal{O}_{\mathrm{v}_{2}}, p_{\text {merge }}, t^{\prime}\right)$ evaluates to true (see Def. 12), and it is not possible to shift $v_{1}$ in $\mathcal{O}_{\mathrm{v}_{1}}\left(t^{\prime}\right)$ so that it is in front of $\mathrm{v}_{2}$ in $\mathcal{O}_{\mathrm{v}_{2}}\left(t^{\prime}\right)$. Since $\mathrm{v}_{2}$ certainly precedes $\mathrm{v}_{1}$ at time $t^{\prime}, \mathrm{v}_{2}$ is the preceding vehicle $\forall t: t^{\prime} \leq t \leq \hat{t}$. Using Def. 13, Prop. 2 follows. The uncertainty of $\hat{t}$ originates from the unspecified road network after the lane merge.

Consequently, at $t_{\text {merge }}$, we can formally guarantee for the first time that $v_{1}$ cannot precede $v_{2}$ in the merging lanes, no matter what vehicle $v_{2}$ is doing (see Fig. 5). Note that before $t_{\text {merge }}$, we cannot eliminate the possibility that $\mathrm{v}_{1}$ can precede $\mathrm{v}_{2}$ in the future.

We sort vehicles in merging lanes by determining $t_{\text {merge }}$ by evaluating PASSED_MERGE $\left(\mathcal{O}_{\mathrm{v}_{1}}, \mathcal{O}_{\mathrm{v}_{2}}, p_{\text {merge }}, \tau_{k}\right)$ for all vehicles pairwise and for all $\tau_{k} \subseteq\left[t_{0}, t_{f}\right]$. If the time $t_{\text {merge }}$ exists, the vehicles $v_{1}$ and $v_{2}$ can be included in the list [v] as $\left(\mathrm{v}_{1}, \mathrm{v}_{2}\right)^{\left[t_{\mathrm{merg}}, \hat{t}\right]}$. Otherwise, we omit the currently compared vehicles in $[\mathrm{v}]$, since it is not possible to determine that $\mathrm{v}_{1}$ cannot precede $\mathrm{v}_{2}$ for any $\tau_{k} \subseteq\left[t_{0}, t_{f}\right]$.

Please note that we do not include length $\mathrm{v}_{2}$ in Def. 10 to determine whether vehicle $\mathrm{v}_{1}$ can precede $\mathrm{v}_{2}$ in forking lanes (unlike Def. 12 for merging lanes), since the earliest point where $\mathrm{v}_{1}$ can possibly pass $\mathrm{v}_{2}$ on its left or right side depends much on the lane geometry. To obtain a simple and provable over-approximative solution, we choose $p_{\text {fork }}$.

\section{Remove Unreachable Occupancies}

After sorting the vehicles in the same lane (for the vehicles where an order can be determined), we remove the occupancy regions of each following vehicle $v_{1}$ which are not reachable due to the preceding vehicle $\mathrm{v}_{2}$. The function TRIMREACHABLE() of Alg. 1 trims the reachable occupancy as shown in Fig. 6: The occupancy $\mathcal{O}_{\mathrm{v}_{1}}(t)$ is shortened such that it is not ahead of the trim line, i.e. after trimming it holds that $\max \left(\mathrm{u}_{\mathcal{O}_{\mathrm{v}_{1}}}(t)\right)=\max \left(\mathrm{u}_{\mathcal{O}_{\mathrm{v}_{2}}}(t)\right)-\operatorname{length}_{\mathrm{v}_{2}}$.

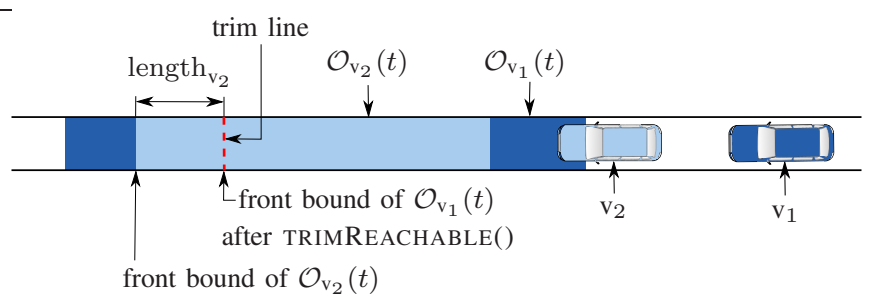

Fig. 6. Removing unreachable occupancies of the following vehicle $\mathrm{v}_{1}$.

\section{NumERICAL EXAMPLES}

We demonstrate our interaction-aware occupancy prediction in hand-crafted scenarios from the CommonRoad benchmarks ${ }^{1}$ [30]. Each benchmark has a unique ID, which are mentioned later. For the sake of clarity, we have so far extracted only two other vehicles, $\mathrm{v}_{1}$ and $\mathrm{v}_{2}$, besides the ego vehicle (and a static obstacle in Scenario I). All results are obtained first by independently predicting the occupancies of $\mathrm{v}_{1}$ and $\mathrm{v}_{2}$ using our tool SPOT ${ }^{2}$ [13] and then by considering their dependencies as described in Alg. 1. In order to evaluate the benefit for the ego vehicle, we compute its drivable area as presented in [31] for the occupancies without considering interaction (case A) and with considering interaction (case B). The drivable area is the area which a vehicle can reach without causing a collision.

Tab. II lists the parameters of the numerical examples, in which we use different initial velocities for the following and preceding vehicle (i.e. $v_{\mathrm{v}_{1,0}} \neq v_{\mathrm{v}_{2,0}}$ ). To evaluate different road conditions, we vary the values for the maximum acceleration $a_{\max }$, which are obtained by choosing a friction coefficient of $\mu=1.0$ and $\mu=0.82$ for a dry, good road, and $\mu=0.25$ for a road covered with snow (and a gravity constant of $g=9.81 \mathrm{~m} / \mathrm{s}^{2}$ ) [32]. We use a time step size of $\Delta t=0.1 \mathrm{~s}$ and a prediction horizon of $t_{f}=2.3 \mathrm{~s}$ for Scenario III and $t_{f}=5.0 \mathrm{~s}$ for the other scenarios.

\footnotetext{
${ }^{1}$ commonroad.in.tum.de

${ }^{2}$ spot.in.tum.de
} 
In all following figures, the following vehicle $\mathrm{v}_{1}$ and the preceding vehicle $\mathrm{v}_{2}$ are depicted in blue and green, respectively. Their predicted occupancies are plotted in their vehicle color and such that the shorter occupancy region is on top of the other one. For Scenarios I and II, the occupancies $\mathcal{O}_{\mathrm{v}}(t)$ are shown for the entire prediction interval, i.e. $t \in$ $\left[t_{0}, t_{f}\right]$, while we set $t \in\left[\left(t_{f}-\Delta t\right), t_{f}\right]$ for Scenarios III and IV. We mark the initial state of the ego vehicle at $t=t_{0}$ with a red circle and its drivable area at $t=t_{f}$ with a red region.

TABLE II

PARAMETERS FOR THE SCENARIOS (S.) I TO IV

\begin{tabular}{llllll}
\hline Parameter & S. Ia & S. Ib & S. II & S. III & S. IV \\
\hline$v_{\mathrm{v}_{1,0}}$ & $28 \mathrm{~m} / \mathrm{s}$ & $28 \mathrm{~m} / \mathrm{s}$ & $14.0 \mathrm{~m} / \mathrm{s}$ & $14.0 \mathrm{~m} / \mathrm{s}$ & $14.0 \mathrm{~m} / \mathrm{s}$ \\
$v_{\mathrm{v}_{2,0}}$ & $8.3 \mathrm{~m} / \mathrm{s}$ & $8.3 \mathrm{~m} / \mathrm{s}$ & $0 \mathrm{~m} / \mathrm{s}$ & $10.0 \mathrm{~m} / \mathrm{s}$ & $6.0 \mathrm{~m} / \mathrm{s}$ \\
$v_{\mathrm{v}_{\mathrm{ego}, 0}}$ & $18.0 \mathrm{~m} / \mathrm{s}$ & $18.0 \mathrm{~m} / \mathrm{s}$ & $14.0 \mathrm{~m} / \mathrm{s}$ & - & $14.0 \mathrm{~m} / \mathrm{s}$ \\
$v_{\mathrm{v}_{1, \max }}$ & $28.0 \mathrm{~m} / \mathrm{s}$ & $28.0 \mathrm{~m} / \mathrm{s}$ & $14.0 \mathrm{~m} / \mathrm{s}$ & $28.0 \mathrm{~m} / \mathrm{s}$ & $14.0 \mathrm{~m} / \mathrm{s}$ \\
$v_{\mathrm{v}_{2, \max }}$ & $17.0 \mathrm{~m} / \mathrm{s}$ & $17.0 \mathrm{~m} / \mathrm{s}$ & $14.0 \mathrm{~m} / \mathrm{s}$ & $28.0 \mathrm{~m} / \mathrm{s}$ & $14.0 \mathrm{~m} / \mathrm{s}$ \\
$v_{\mathrm{vego}_{\max }}$ & $28.0 \mathrm{~m} / \mathrm{s}$ & $28.0 \mathrm{~m} / \mathrm{s}$ & $14.0 \mathrm{~m} / \mathrm{s}$ & - & $14.0 \mathrm{~m} / \mathrm{s}$ \\
$a_{\max }$ & $8.0 \mathrm{~m} / \mathrm{s}^{2}$ & $2.5 \mathrm{~m} / \mathrm{s}^{2}$ & $2.5 \mathrm{~m} / \mathrm{s}^{2}$ & $10.0 \mathrm{~m} / \mathrm{s}^{2}$ & $2.5 \mathrm{~m} / \mathrm{s}^{2}$ \\
\hline
\end{tabular}

\section{A. Two-Lane Road (Scenario I)}

Scenario I (CommonRoad ID: S=GER_B471_1a) features a rural road with one lane per driving direction and a static obstacle (displayed as a gray box) in the lane of the ego vehicle, as shown in Fig. 7. Thus, the ego vehicle requires an overtaking maneuver but also has to avoid a collision with the two oncoming vehicles. Fig. 8 shows the predicted occupancies of $v_{1}$ and $v_{2}$ in Scenario Ia. The occupancies of the following vehicle $v_{1}$ (shown in blue) reach in front of the slower preceding truck $\mathrm{v}_{2}$ (shown in green) (see Fig. 8(a)). As mentioned before, we plot the occupancy sets such that the shorter occupancy is on top of the other one. The result of removing the unreachable occupancy regions after sorting the two vehicles in the same current lane is shown in Fig. 8(b). It can be seen that the difference is not much more than the length of the preceding truck. In Scenario Ib, we use $a_{\max }=2.5 \mathrm{~m} / \mathrm{s}^{2}$ and plot the occupancy sets in Fig. 9. When comparing case A in Fig. 9(a) and case B in Fig. 9(b), it can be observed that the effect of the interaction is significant and greater than in Scenario Ia.

In Fig. 10, the benefit for the ego vehicle from the interaction-aware prediction is evaluated using the drivable area of the ego vehicle under the given velocity and acceleration limits (see Tab. II). Since no drivable area exists in front of the static obstacle in case A, overtaking is only safely possible when removing unreachable occupancies (case B).

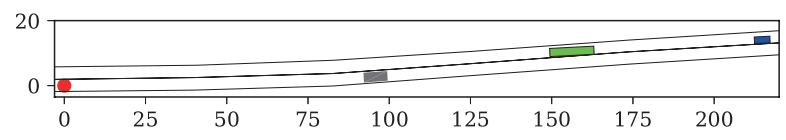

Fig. 7. Initial configuration of Scenario I.

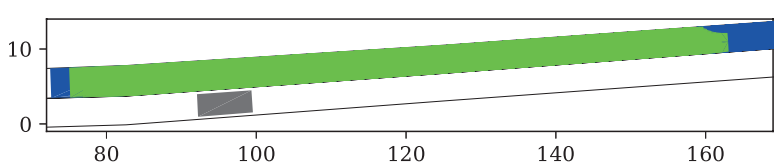

(a) Case A: not considering interaction.

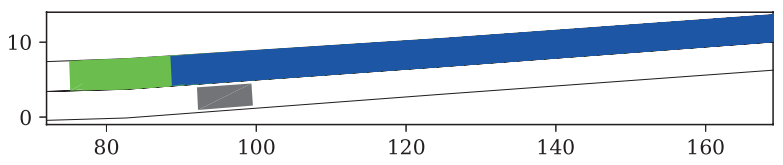

(b) Case B: considering interaction.

Fig. 8. Occupancies in Scenario Ia $\left(a_{\max }=8.0 \mathrm{~m} / \mathrm{s}^{2}\right)$.

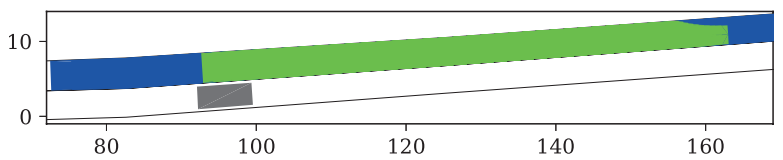

(a) Case A: not considering interaction.

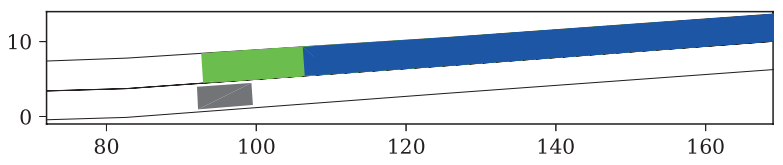

(b) Case B: considering interaction.

Fig. 9. Occupancies in Scenario Ib $\left(a_{\max }=2.5 \mathrm{~m} / \mathrm{s}^{2}\right)$.

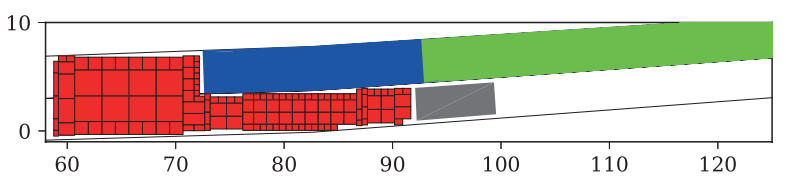

(a) Case A: not considering interaction.

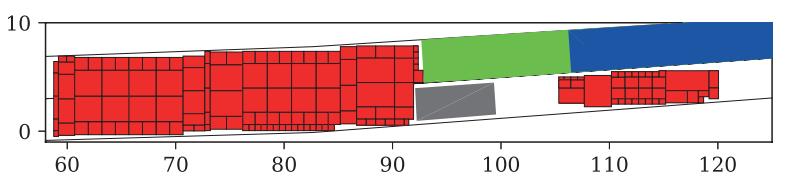

(b) Case B: considering interaction.

Fig. 10. Drivable area of the ego vehicle in Scenario Ib.

\section{B. Intersection of Multiple Two-Lane Roads (Scenario II)}

Scenario II presents an urban intersection, where four twolane roads cross (CommonRoad ID: S=GER_Ffb_1b). As depicted in Fig. 11, the two vehicles $\mathrm{v}_{1}$ and $\mathrm{v}_{2}$ are driving south, while the ego vehicle is approaching the intersection from east. The independently predicted occupancy sets are plotted in Fig. 12(a) together with the drivable area of the ego vehicle. Fig. 12(b) shows the trimmed occupancies after removing the unreachable area of $v_{1}$. It can be seen that when considering interaction in the occupancy prediction, the ego vehicle can safely cross the intersection. At time $t_{f}$, the difference of the drivable area between cases A and B is larger than $100 \mathrm{~m}^{2}$. Please note that in this intersection scenario, which consists of multiple road forks, we can sort the vehicles to consider their interaction, since the occupancy of the preceding vehicle $\mathrm{v}_{2}$ does not split onto several lanes yet, i.e. NOT_PASSED_FORK $\left(\mathcal{O}_{\mathrm{v}_{2}}, p_{\text {fork }}, t_{f}\right)$ evaluates to true. 


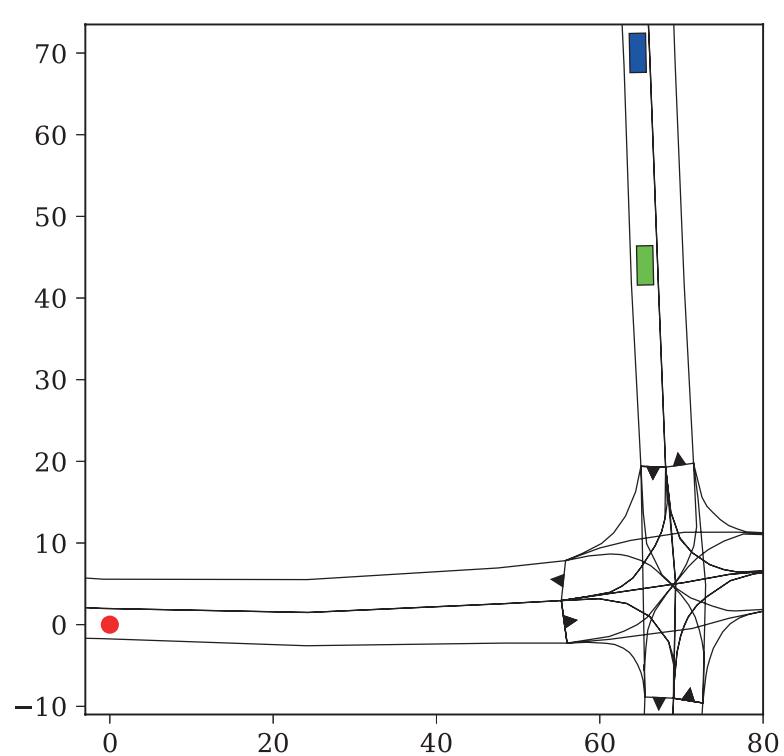

Fig. 11. Initial configuration of Scenario II.

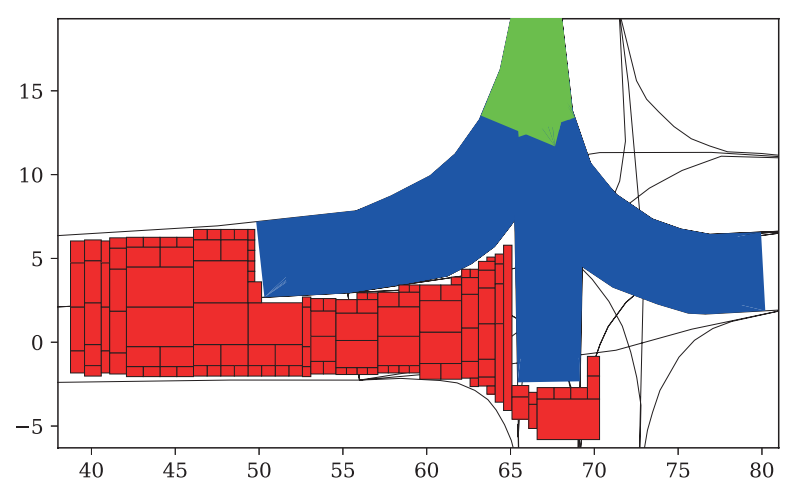

(a) Case A: not considering interaction.

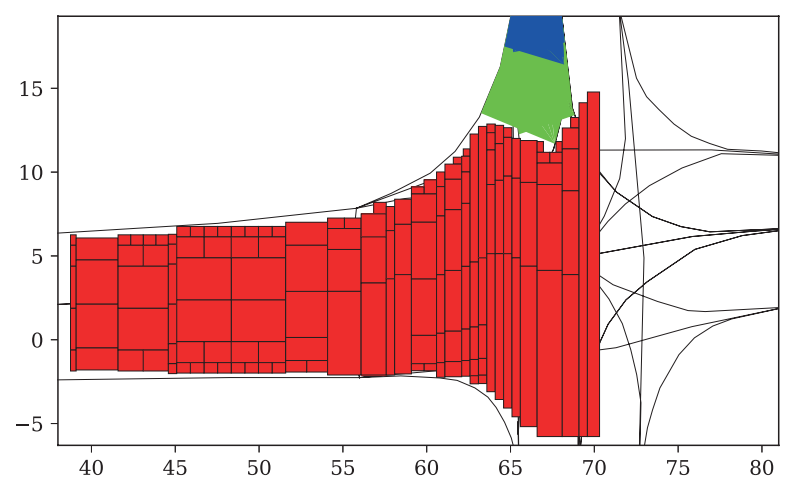

(b) Case B: considering interaction.

Fig. 12. Drivable area of the ego vehicle in Scenario II.

\section{Road with Merging Lanes (Scenario III)}

In Scenario III, we demonstrate the sorting of vehicles in merging lanes (CommonRoad ID: S=Z_Merge_1a). As shown in Fig. 13, one vehicle is driving in each of the merging lanes. Their occupancy sets are plotted for $t \in\left[\left(t_{f}-\Delta t\right), t_{f}\right]$, where $t_{f}=2.3 \mathrm{~s}$. Since the green vehicle $\mathrm{v}_{2}$ will definitely precede the blue vehicle $\mathrm{v}_{1}$ (as $t \geq t_{\text {merge }}$ ), we can remove the unreachable occupancy region of $\mathrm{v}_{1}$ (see Fig. 13(b)).

\section{Multi-Lane Road (Scenario IV)}

As mentioned in Sec. IV, considering interaction is not beneficial in multi-lane roads, which we illustrate with the following example (CommonRoad ID: S=GER_Muc_2b). Fig. 14 shows two lanes with the same driving direction, where two vehicles $v_{1}$ and $v_{2}$ are driving in the right lane and the ego vehicle in the left lane. It can be seen that considering the vehicles pairwise for determining the interactions is not sufficient, since the following vehicle $\mathrm{v}_{1}$ might be blocked by two vehicles at once: the preceding vehicle $\mathrm{v}_{2}$ and the ego vehicle. One might be able to remove small unreachable regions of the following vehicle's occupancies, yet that region is also occupied by the preceding vehicle. Thus, we gain no benefit for the drivable area of the ego vehicle. Moreover, in contrast to two-lane roads, vehicles can easily overtake each other on multi-lane roads and hence almost all occupancy regions are reachable. For this reason, we have not extended our method to multi-lane roads.

\section{DISCUSSION}

The numerical examples show that considering interaction between vehicles in the same lane increases the solution space of the ego vehicle. However, removing unreachable occupancies only shows substantial benefit in some cases, e.g. only for bad weather conditions (i.e. low values of $\left.a_{\max }\right)$ or certain configurations of initial states. Thus, we suggest applying our approach selectively. The occupancy sets of all surrounding vehicles should always be predicted independently first. In the remaining computation time during online execution, one can refine the over-approximative prediction by trimming reachable occupancy regions. Due to the anytime property of our algorithm, one can terminate it when computation time is required elsewhere.

We remove unreachable occupancy regions under the assumption that a vehicle does not change to a lane with

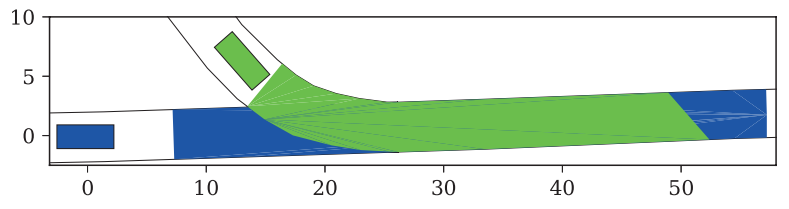

(a) Case A: not considering interaction.

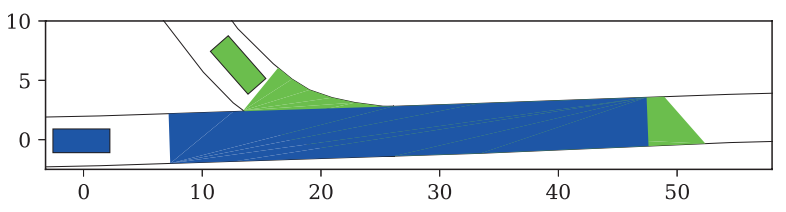

(b) Case B: considering interaction.

Fig. 13. Initial configuration and occupancies for $t \in\left[\left(t_{f}-\Delta t\right), t_{f}\right]$ in Scenario III.

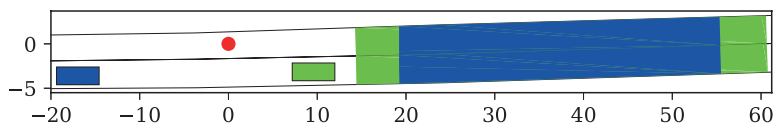

Fig. 14. Initial configuration and occupancies for $t \in\left[\left(t_{f}-\Delta t\right), t_{f}\right]$ in Scenario IV. 
the opposite driving direction (constraint $C_{\text {lane }}$ of Tab. I). However, as described in the constraint management in [13], we immediately remove $C_{\text {lane }}$ if it becomes violated. Thus, the set-based prediction considers the occupancy of the vehicle in its new lane, as well as in front of a preceding vehicle, which might have been removed by our interactionaware method before the lane change.

\section{CONClusion And Future Work}

For the first time, we consider interaction between vehicles in set-based prediction. Our formal approach removes unreachable occupancy regions of vehicles in the same lane by sorting all vehicles and determining unreachable areas. The benefits of our anytime algorithm are demonstrated in numerical experiments based on scenarios from the CommonRoad benchmark repository. Since the drivable area of the ego vehicle is larger in all scenarios when interaction is considered, this work improves the quality of the overapproximative occupancy prediction and increases the safe solution space for the ego vehicle.

Future work contains further experiments on different scenarios. In addition, we wish to include interaction between traffic participants at intersections when considering applicable traffic rules.

\section{ACKNOWLEDGMENT}

The authors thank Hannes Bibel for his valuable contribution to developing our approach and Vanessa Bui for creating numerical examples. This work was founded by the BMW Group within the CAR@TUM project.

\section{REFERENCES}

[1] S. Lefèvre, D. Vasquez, and C. Laugier, "A survey on motion prediction and risk assessment for intelligent vehicles," ROBOMECH Journal, vol. 1, no. 1, pp. 1-14, 2014.

[2] A. Barth and U. Franke, "Where will the oncoming vehicle be the next second?" in Proc. of the IEEE Intelligent Vehicles Symposium, 2008, pp. 1068-1073.

[3] A. Eidehall, "Multi-target threat assessment for automotive applications," in Proc. of the 14th International IEEE Conference on Intelligent Transportation Systems, 2011, pp. 433-438.

[4] M. Brännström, E. Coelingh, and J. Sjöberg, "Model-based threat assessment for avoiding arbitrary vehicle collisions," IEEE Transactions on Intelligent Transportation Systems, vol. 11, no. 3, pp. 658-669, 2010.

[5] J.-H. Kim and D.-S. Kum, "Threat prediction algorithm based on local path candidates and surrounding vehicle trajectory predictions for automated driving vehicles," in Proc. of the IEEE Intelligent Vehicles Symposium, 2015, pp. 1220-1225.

[6] J. Wei, J. M. Snider, T. Gu, J. M. Dolan, and B. Litkouhi, "A behavioral planning framework for autonomous driving," in Proc. of the IEEE Intelligent Vehicles Symposium, 2014, pp. 458-464.

[7] A. Eidehall and L. Petersson, "Statistical threat assessment for general road scenes using Monte Carlo sampling," IEEE Transactions on Intelligent Transportation Systems, vol. 9, pp. 137-147, 2008.

[8] T. Gindele, S. Brechtel, and R. Dillmann, "Learning driver behavior models from traffic observations for decision making and planning," IEEE Intelligent Transportation Systems Magazine, vol. 7, no. 1, pp. 69-79, 2015.

[9] A. Lambert, D. Gruyer, G. S. Pierre, and A. N. Ndjeng, "Collision probability assessment for speed control," in Proc. of the 11th International IEEE Conference on Intelligent Transportation Systems, 2008, pp. 1043-1048.

[10] M. Althoff, O. Stursberg, and M. Buss, "Model-based probabilistic collision detection in autonomous driving," IEEE Transactions on Intelligent Transportation Systems, vol. 10, no. 2, pp. 299-310, 2009.
[11] M. Althoff and A. Mergel, "Comparison of Markov chain abstraction and Monte Carlo simulation for the safety assessment of autonomous cars," IEEE Transactions on Intelligent Transportation Systems, vol. 12, no. 4, pp. 1237-1247, 2011.

[12] M. Althoff and S. Magdici, "Set-based prediction of traffic participants on arbitrary road networks," IEEE Transactions on Intelligent Vehicles, vol. 1, no. 2, pp. 187-202, 2016.

[13] M. Koschi and M. Althoff, "SPOT: A tool for set-based prediction of traffic participants," in Proc. of the IEEE Intelligent Vehicles Symposium, 2017, pp. 1679-1686.

[14] S. Magdici and M. Althoff, "Fail-safe motion planning of autonomous vehicles," in Proc. of the 19th IEEE International Conference on Intelligent Transportation Systems, 2016, pp. 452-458.

[15] D. Georgiev, P. T. Kabamba, and D. M. Tilbury, "A new model for team optimization: The effects of uncertainty on interaction," IEEE Transactions on Systems, Man and Cybernetics - Part A: Systems and Humans, vol. 38, no. 6, pp. 1234-1247, 2008.

[16] E. Käfer, C. Hermes, C. Woehler, H. Ritter, and F. Kummert, "Recognition of situation classes at road intersections," in Proc. of the IEEE International Conference on Robotics and Automation, 2010, pp. 3960-3965.

[17] A. Lawitzky, D. Althoff, C. F. Passenberg, G. Tanzmeister, D. Wollherr, and M. Buss, "Interactive scene prediction for automotive applications," in Proc. of the IEEE Intelligent Vehicles Symposium, 2013, pp. 1028-1033.

[18] M. Althoff, O. Stursberg, and M. Buss, "Safety assessment of driving behavior in multi-lane traffic for autonomous vehicles," in Proc. of the IEEE Intelligent Vehicles Symposium, 2009, pp. 893-900.

[19] N. Oliver and A. P. Pentland, "Graphical models for driver behavior recognition in a smartcar," in Proc. of the IEEE Intelligent Vehicles Symposium, 2000, pp. 7-12.

[20] M. Liebner, C. Ruhhammer, F. Klanner, and C. Stiller, "Driver intent inference at urban intersections using the intelligent driver model," in Proc. of the IEEE Intelligent Vehicles Symposium, 2012, pp. 11621167.

[21] T. Gindele, S. Brechtel, and R. Dillmann, "A probabilistic model for estimating driver behaviors and vehicle trajectories in traffic environments," in Proc. of the 13th International IEEE Conference on Intelligent Transportation Systems, 2010, pp. 1625-1631.

[22] G. Agamennoni, J. I. Nieto, and E. M. Nebot, "Estimation of multivehicle dynamics by considering contextual information," IEEE Transactions on Robotics, vol. 28, no. 4, pp. 855-870, 2012.

[23] W. Yao, Q. Zeng, Y. Lin, D. Xu, H. Zhao, F. Guillemard, S. Géronimi, and F. Aioun, "On-road vehicle trajectory collection and scenebased lane change analysis: Part II," IEEE Transactions on Intelligent Transportation Systems, vol. 18, no. 1, pp. 206-220, 2017.

[24] S. Klingelschmitt, F. Damerow, V. Willert, and J. Eggert, "Probabilistic situation assessment framework for multiple, interacting traffic participants in generic traffic scenes," in Proc. of the IEEE Intelligent Vehicles Symposium, 2016, pp. 1141-1148.

[25] S. Lefèvre, C. Laugier, and J. Ibañez-Guzmán, "Evaluating risk at road intersections by detecting conflicting intentions," in Proc. of the IEEE/RSJ International Conference on Intelligent Robots and Systems, 2012, pp. 4841-4846.

[26] P. Bender, J. Ziegler, and C. Stiller, "Lanelets: Efficient map representation for autonomous driving," in Proc. of the IEEE Intelligent Vehicles Symposium, 2014, pp. 420-425.

[27] M. Althoff and J. M. Dolan, "Online verification of automated road vehicles using reachability analysis," IEEE Transactions on Robotics, vol. 30, no. 4, pp. 903-918, 2014.

[28] United Nations Economic Commission for Europe, "Vienna convention on road traffic," United Nations, 1968.

[29] A. Rizaldi and M. Althoff, "Formalising traffic rules for accountability of autonomous vehicles," in Proc. of the 18th IEEE International Conference on Intelligent Transportation Systems, 2015, pp. 16581665

[30] M. Althoff, M. Koschi, and S. Manzinger, "CommonRoad: Composable benchmarks for motion planning on roads," in Proc. of the IEEE Intelligent Vehicles Symposium, 2017, pp. 719-726.

[31] S. Söntges and M. Althoff, "Computing the drivable area of autonomous road vehicles in dynamic road scenes," IEEE Transactions on Intelligent Transportation Systems, [to appear].

[32] C.-G. Wallman and H. Åström, "Friction measurement methods and the correlation between road friction and traffic safety," in VTI meddelande. Swedish National Road and Transport Research Institute, 2001. 